\title{
Experimental investigation of dispersion phenomenon in a fractured porous medium
}

\author{
Ali Sanati $^{1}$, Mohammad Yousefi Khoshdaregi ${ }^{2}$ \\ ${ }^{I}$ Faculty of Petroleum and Petrochemical Engineering, Hakim Sabzevari University, Sabzevar, Iran \\ ${ }^{2}$ Department of Petroleum Engineering, Petroleum University of Technolog, Ahvaz, Iran \\ *Corresponding author E-mail: sanatiam@gmail.com
}

Copyright $\odot 2015$ Ali Sanati, Mohammad Yousefi Khoshdaregi. This is an open access article distributed under the Creative Commons Attribution License, which permits unrestricted use, distribution, and reproduction in any medium, provided the original work is properly cited.

\begin{abstract}
Dispersion of fluids flowing through porous media is an important phenomenon in miscible displacement. Dispersion causes instability of miscible displacement flooding; therefore, to obtain and maintain the miscibility zone, the porous medium dispersivity should be considered in displacing fluid volume calculation. Many works have been carried out to investigate the dispersion phenomenon in porous media in terms of theory, laboratory experiments and modeling. What is still necessary is to study the effects of presence of fracture in a porous medium on dispersion coefficient or dispersivity. In this work dispersion phenomenon in a fractured porous medium has been investigated through a series of miscible displacement tests on homogeneous sandstone core samples. Tests were repeated on the same core samples with induced fracture in the flow direction. The effects of fracture on miscible displacement flooding have been studied by comparison of the results of dispersion tests in the absence and presence of fracture. In the presence of fracture, breakthrough time reduced and the tail of effluent $\mathrm{S}$-shaped curve smeared. Moreover, the slope of S-shaped curve at 1 pore volume of injected fluid was lower than homogeneous case which means dispersion coefficient increased. The results presented in this work provide an insight to the understanding of dispersion phenomenon for modeling of miscible displacement process through naturally fractured reservoirs.
\end{abstract}

Keywords: Enahanced Oil Recovery; Dispersion; Miscible Displacement; Fracture.

\section{Introduction}

In most of the enhanced recovery processes such as miscible drives, carbon dioxide flooding, as well as other recovery methods, mixing of two miscible fluids in a porous medium plays a very important role.[1] Many studies have been devoted to mechanics of miscible displacement, focusing on the longitudinal and transverse dispersion. $[2,3,4,5,6]$ As one miscible fluid displaces another, the displacing solution continuously mixes with the resident fluid, so that the arrival of the displacing solution at a given point in the porous medium is characterized by a gradual change in the solution concentration from that of the original fluid to that of the invading fluid. This mixing or interfusing of the two fluids, due to both molecular diffusion and convection, is termed as dispersion. Dispersion theory is important in the study of the miscible recovery of oil, movement of trace contaminants such as radioactive waste and heavy metals, Infiltration of saltwater in groundwater systems, chromatography, fluid- solid catalytic and non-catalytic reactions, etc.[5]

\section{Materials and methods}

The experimental apparatus used for this study was designed to isolate and study the effect of fracture on dispersion phenomena.(Figure1) In order to isolate the dispersion phenomena due to fracture, several interfering processes had to be eliminated. These processes include adsorption of the displaced and displacing fluid on the surface of the porous medium; and dispersive effects due to viscosity and density differences between the displaced and displacing fluids. 
Two groups of relatively clean sandstone samples were tested. Sandstone was used because of its high permeability and porosity. During the tests, minimum volume of each fluid sample used for RI measurement was about $2 \mathrm{cc}$, so pore volume was a crucial parameter in this study. An increase in length of core samples could supply more pore volume, but some limitations in laboratory apparatuses restricted core samples length to be maximum $15 \mathrm{~cm}$ long. Dimensions of core samples are presented in Tables 1 , and Table 2.

To conduct miscible displacement tests for determining the dispersion coefficient, two fluids are necessary which must meet the requirements such as: completely miscible, equal viscosities and equal densities, wide range of refractive indices, no chemical reactions with rock matrix, no adsorption on rock matrix, easy, and cost effective, easy to clean for repeated runs and nonvolatile.

Based on these considerations, Naphtha and Gasoil were selected. These two fluids meet all the above requirements except their viscosities. So naphtha with lower viscosity was used as displaced fluid, while gasoil with higher viscosity was used as displacing fluid to avoid viscose fingering during tests.

Table 1: Dimensions of Group No.1 Core Samples

\begin{tabular}{llll}
\hline Sample No. & Type & Diameter $(\mathrm{mm})$ & Length $(\mathrm{mm})$ \\
\hline No. 1-1 & Sandstone & 37.63 & 43.01 \\
No. 1-2 & Sandstone & 37.63 & 37.30 \\
No. 1-3 & Sandstone & 37.63 & 36.33 \\
No. 1-4 & Sandstone & 37.63 & 36.21 \\
Total & Sandstone & 37.63 & 152.85 \\
\hline
\end{tabular}

Table 2: Dimensions of Group No.2 Core Samples

\begin{tabular}{llll}
\hline Sample No. & Type & Diameter $(\mathrm{mm})$ & Length $(\mathrm{mm})$ \\
\hline No. 2-1 & Sandstone & 37.78 & 35.05 \\
No. 2-2 & Sandstone & 37.78 & 36.33 \\
No. 2-3 & Sandstone & 37.78 & 41.83 \\
No. 2-4 & Sandstone & 37.78 & 35.21 \\
Total & Sandstone & 37.78 & 148.42 \\
\hline
\end{tabular}

\subsection{Expirimental procedure}

Different experimental procedures and techniques were required in this investigation. The experimental procedure for this study is divided into two parts. Part one is the experiment on conventional sandstone core samples, and part two is the same experiment on fractured core samples. All core samples were vacuumed and saturate with gasoil. After that, their pore volume and permeability were measured using core flood apparatus. The results of pore volume and permeability measurements are shown in Table 3.

Table 3: Pore Volume and Permeability of Each Group of Core Samples

\begin{tabular}{lll}
\hline Group No. & No.1 & No.2 \\
\hline Pore volume (cc) before Fracturing & 26.26 & 30.35 \\
Pore volume (cc) after Fracturing & 25.38 & 29.83 \\
Permeability (md) before Fracturing & 133 & 179 \\
Permeability (md) after Fracturing & 195 & 397 \\
\hline
\end{tabular}

To conduct the dispersion measurements on core samples, a miscible displacement test is so designed that an s-shaped concentration profile can be obtained. For the miscible displacement tests, two major pieces of equipment required are (1) a constant-rate pump for fluid injection, and (2) a refractometer for measuring the effluent concentration. If the refractive indices of two fluids are known, their percentage in a mixture can be determined from the measured refractive index for this mixture. Therefore, it is necessary to calibrate this device for the selected fluids for miscible displacements (Gasoil and Naphtha). The calibration procedure is to measure the refractive indices of mixtures with a variety of percentages of the two fluids such as $0 / 100,10 / 90,20 / 80$, and so on until 100/0. The relationship between fluid percentage and refractive index shows a straight line for gasoil and naphtha as shown in Figure 2. From this plot, measuring the refractive index of a mixture, the percentages of the components can be easily found. 


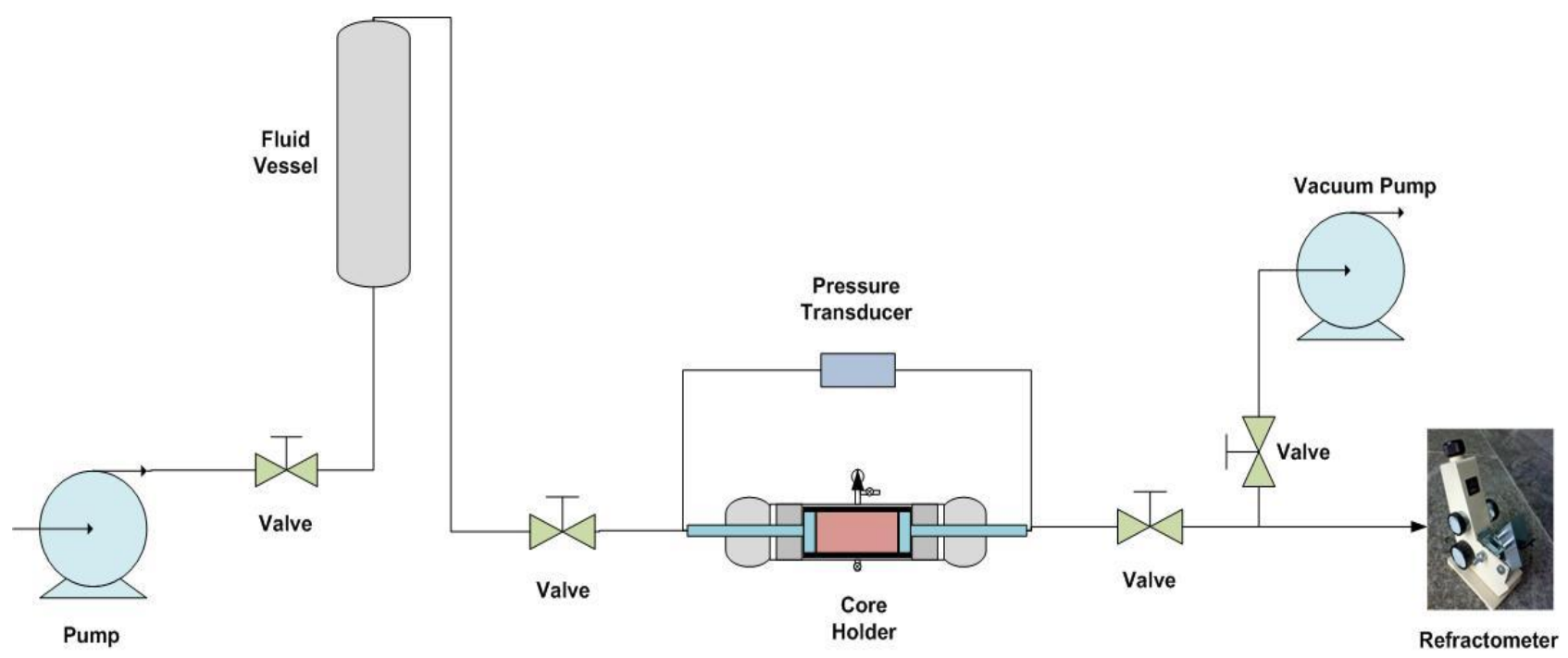

Fig. 1: A Schematic Diagram of System Setup

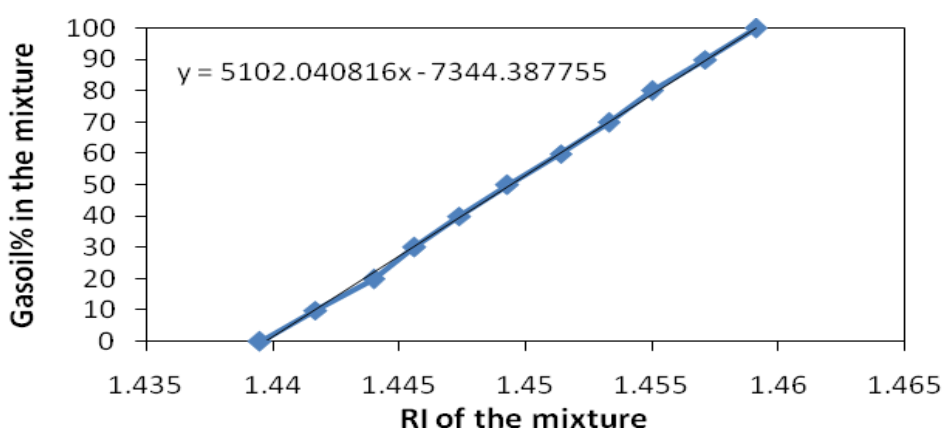

Fig. 2: Calibration Curve

Except the favorable viscosities and densities of the two fluids, an appropriate flow rate for the miscible displacement tests is also critical to obtain a unique value of dispersivity. The flow rate of $1 \mathrm{cc} / \mathrm{min}$ was selected for conducting dispersion tests. Based on the above description, the procedure of conducting a miscible displacement can be described below:

At the end of permeability measurement section, the pump injects displaced fluid (naphtha) at $1 \mathrm{cc} / \mathrm{min}$ flow rate until the pressure drop stabilized. Then both inlet and outlet valves of core holder were closed at the same time to keep the pressure of fluid inside core holder constant. After that, fluid vessel and all connection lines were cleaned and filled with displacing fluid (gasoil). Pump started to inject gasoil at $1 \mathrm{cc} / \mathrm{min}$ into the core holder, but both valves were closed. When the injection pressure reached the pressure inside core holder, both valves were opened and at the same time, timer started.

The effluent fluid was collected using sampling tubes and the time of switching tubes was recorded. Each tube collected 1 to $4 \mathrm{cc}$ of fluid flowing out of core holder related to the time of sampling. Sampling intervals at the start and end of displacement test were longer than sampling intervals in the middle of test. The refractive index of each sample was analyzed using a refractometer, while the concentration was calculated using calibration curves. Once the effluent fluid consisted entirely of the displacing fluid, the test was terminated.

\section{Results}

As mentioned before, naphtha and gasoil were used as displaced and displacing fluids respectively. Results of dispersion tests on each group of core samples are shown in Figure 3 and Figure 4 . In these two Figures relative concentration versus injected pore volume of displacing fluid is plotted. As can be seen, in two figures relative concentration of 0.5 is achieved at 1 pore volume of injected fluid. This shows that these two core sample groups act like homogeneous porous media. Figure 5 shows dispersion test results of two core sample groups together. These tests conducted on fracture core samples, the same as previous part, and results of dispersion tests on each group of core samples are shown in Figure 6 and Figure 7. In these Figures relative concentration versus injected pore volume of displacing fluid is plotted. Figure 8 shows dispersion test results of two core sample groups together. As can be seen in Figure 8, fracture systems of both groups have similar results. Both of them at one pore volume of injected fluid achieve a "0.6" relative concentration. Also, in Figure 9 and Figure 10 results of dispersion tests on each group of core samples with fracture and without fracture are shown together. Qualitative effect of fracture on dispersion effluent curve can be seen in these two Figures. 


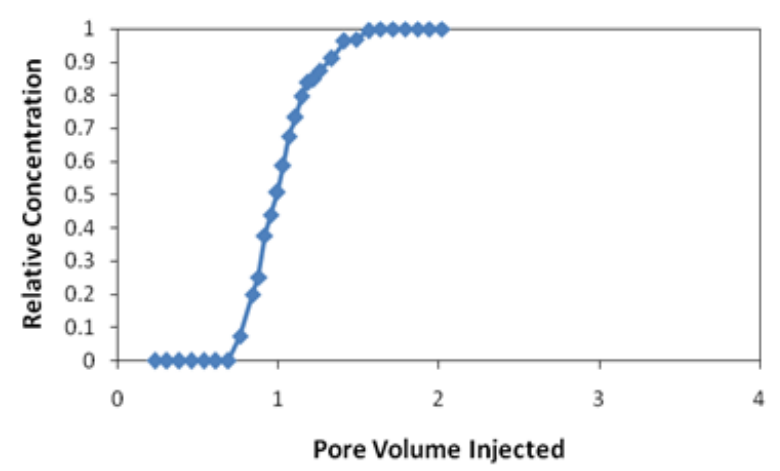

Fig. 3: Dispersion Test on Non-Fractured Core Group No.1

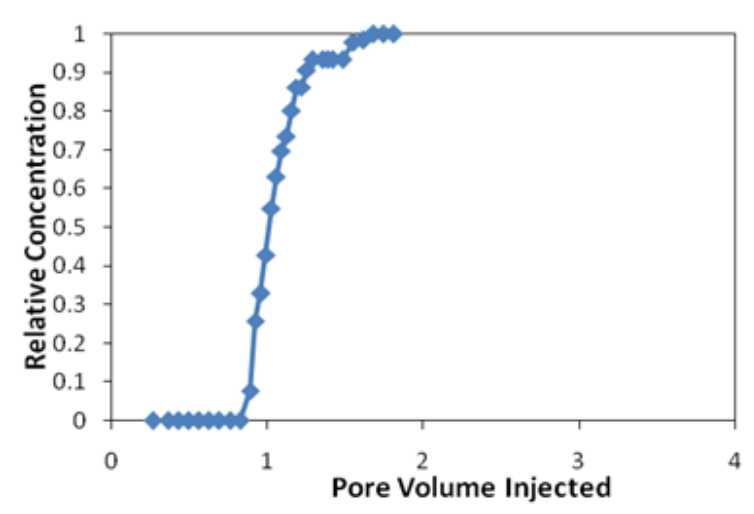

Fig. 4: Non-Fractured Core Group No.2

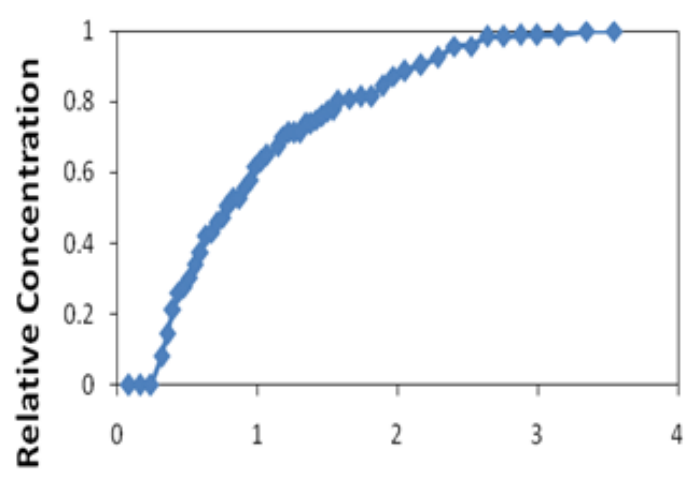

Pore Volume Injected

Fig. 6: Fractured Core Group No.2

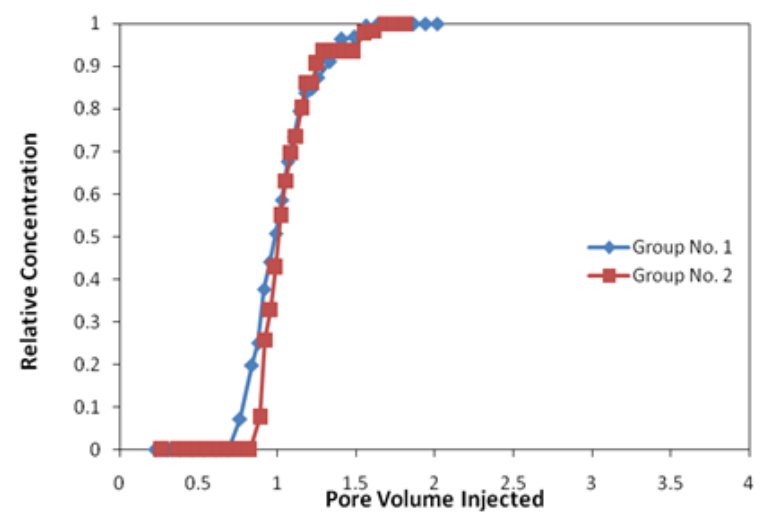

Fig. 5: Non -Fractured Core Sample Group

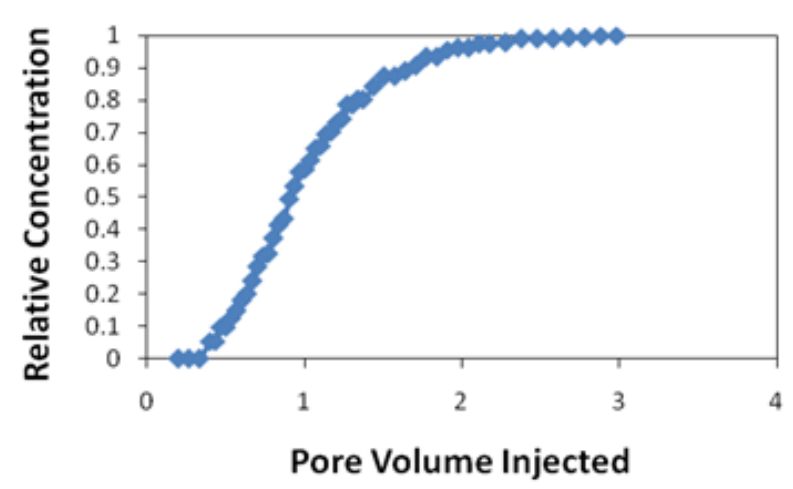

Fig. 7: Fractured Core Group No.1

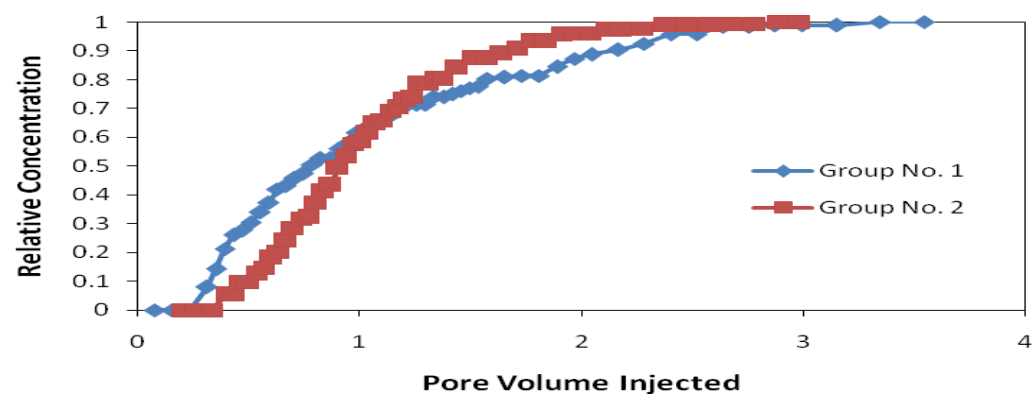

Fig. 8: Dispersion Test on Two Fractured Core Sample Groups 


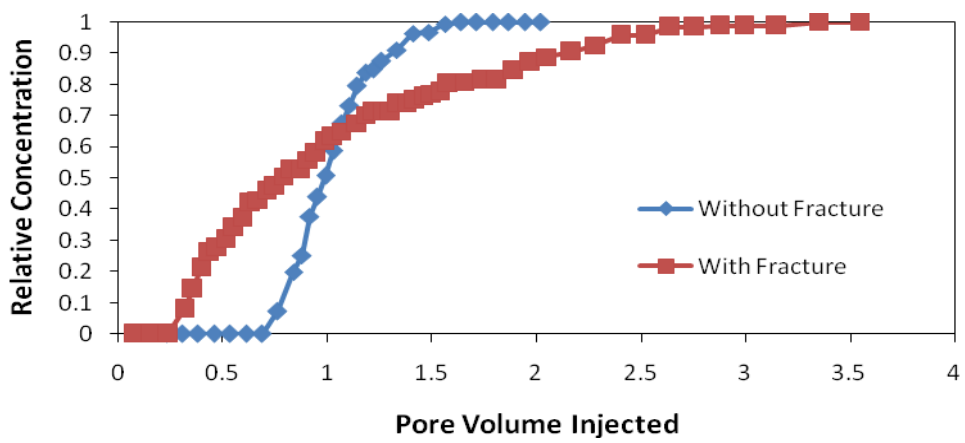

Fig. 9: Comparison of Dispersion Test on Fractured and Non-Fractured Group No.1

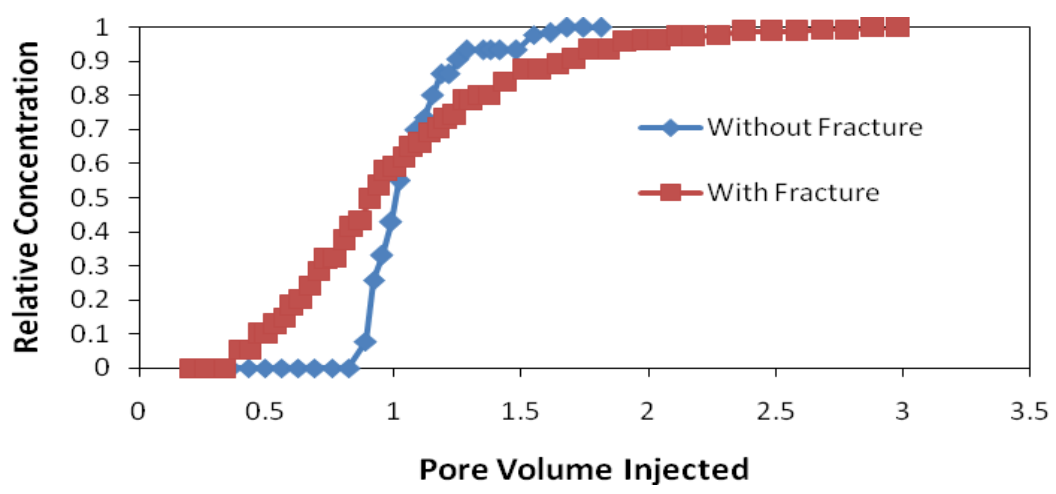

Fig. 10: Comparison Dispersion Test on Fractured and Non-Fractured Group No.2

\section{Conclusion}

The objective of this Thesis is to study the effect of fractures on dispersion during miscible displacement flooding. Based on the results obtained from different experiments, the following conclusions are made:

In a qualitative pint of view, results of tests conducted on conventional porous media is similar to results of a homogeneous porous media in which relative concentration of 0.5 will achieve at one pore volume injection of displacing fluid.

In a qualitative point of view, the induced fracture will reduce breakthrough time. Moreover, for core samples of group No. 1 and group No. 2, near one pore volume injection of displacing fluid, relative effluent concentration is greater than 0.5. Also, $100 \%$ displacement will achieve at higher pore volume injection than the case with nonfractured core samples.

\section{References}

[1] Dutta, S., 1984, an Experimental Investigation of Dispersivity and its Role as an Oil Reservoir Rock Property: MSc Thesis, University of Oklahoma.

[2] Sandrea, R., 1974, Dynamics of Petroleum Reservoirs under Gas Injection: Gulf Publishing Company, Houston, Texas.

[3] Niemann, E.H., Greenkorn R.A., and Eckert, R.E., 1971, Dispersion during Flow in Nonuniform, Heterogeneous Porous Media: SPE Paper No. 3365 .

[4] Chen, S., 1991, Investigation of Dispersivity as a Reservoir Rock Characteristic and its Determination from Well Logs: PhD Thesis, University of Oklahoma.

[5] Fred, I. and Stalkup J, 1984, Miscible Displacement: SPE Monograph Series.

[6] K. M. G. Li, 1983, Random Choice Method for Treating the Convection-Diffusion Equation: SPE Paper No. 12237 